\title{
Brain areas underlying retrieval of nouns and verbs: Grammatical class and task demand effects.
}

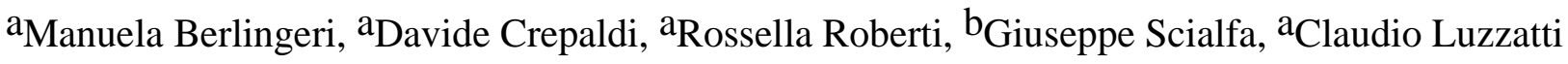 \\ and a Eraldo Paulesu
}

\footnotetext{
a Department of Psychology, University of Milano-Bicocca, Italy

b Neuroradiology Department, Ospedale Niguarda Ca' Granda, Milano
}

\section{Introduction}

Current data on the neural correlates of noun and verb processing are inconsistent as studies using different imaging techniques and/or different tasks have provided remarkably different results. Verbs have been reported to be associated with left frontal activity (Shapiro et al., 2005), with left parietal regions (Martin et al., 1995) and with activation in the inferior temporal regions (Tranel et al., 2005). Furthermore, past neuroimaging evidence highlights a crucial role of the left inferior frontal gyrus in noun processing (Tyler et al., 2003), as well as a crucial role of the inferior temporal (Bedny et al., 2006) and occipito-temporal (Fujimaki et al., 1999) areas. We developed an fMRI study to clarify this issue by assessing task-independent and task-dependent grammatical-classspecific effects during lexical retrieval tasks.

\section{Materials and Methods}

Subjects. Twelve right-handed undergraduate Italian students participated in the study (six females and six males, aged 20 to 34 ).

Tasks and Materials. Subjects retrieved nouns and verbs during two different tasks: a Picture Naming Task (PNT) similar to the task adopted in previous experiments and a Grammatical Class Switching Task (GCST) in which verbs and nouns were retrieved from their corresponding noun or verb (saying vincere (to win) when reading vittoria (victory), or viceversa; see Crepaldi et al., 2006). It should be noted that the typical picture naming task used to assess noun or verb retrieval is necessarily imbalanced for imageability and visual complexity, as pictures for verbs are more complex than pictures for objects; in the GCST nouns and verbs had the same level of imageability. Prior to taking the fMRI scans, the subjects familiarized with the experimental stimuli so that strategic search of targets was minimized; reaction times were recorded for the different tasks during the familiarization sessions. 
fMRI data acquisition and analysis. MRI scans were performed on a 1.5 T Marconi-Philips Infinion Scanner, using an EPI gradient echo sequence (TR: $3 \mathrm{~s}$; TE: $60 \mathrm{~ms}$; Flip angle: 90 $)$. The selected volume consisted of 26 contiguous transverse images (thickness $=5 \mathrm{~mm}$; gap $=0 \mathrm{~mm}$ ), acquired every 3.05 seconds. Each of the tasks described above generated $120 \mathrm{fMRI}$ scans collected in alternating blocks of 10 baseline scans and 10 experimental task scans. Condition-specific effects were estimated according to the general linear model (GLM) in SPM2. The statistical threshold was set at $\mathrm{p}=.001$.

\section{Results}

Behavioural results. RTs for nouns and verbs differed in a task-dependent manner (Figure 1a), so responses took longer for verbs in the PNT and for nouns in the GCST (683 ms vs $640 \mathrm{~ms}$ in the PNT, $609 \mathrm{~ms}$ vs $673 \mathrm{~ms}$ in the GCST; Ftask-by-grammatical-class[1,9]= 54.68; $\mathrm{p}<.001)$. This pattern is in line with the higher task demand required for verb retrieval in the PNT and for noun retrieval in the GCST.

fMRI results. In both experimental tasks, verbs were consistently associated with an increased bilateral activation in the precentral and postcentral gyri, in the right SMA, in the right inferior parietal lobule and, bilaterally, in the paracentral lobule, in the superior parietal lobule and in the precuneus. No consistent network emerged for nouns across tasks. There was also a task-dependent difference between verbs and nouns consistent with the behavioural data, with a greater activation of the left inferior frontal gyrus triggered by verbs in the PNT, and by nouns in the GCST (see Figure 1b).

\section{Discussion}

The systematic involvement of parietal cortices during verb processing suggests an intimate relationship between lexical-semantic and sensory-motor representations for actions. Importantly, the battery also included verbs without a prominent motor component, indicating that verb lexicalsemantic representations are related to action-oriented spatial knowledge even for verbs with a very low degree of actionality.

On the other hand, data show that the involvement of the left inferior frontal gyrus and the left insula in lexical retrieval may not reflect a grammatical class effect (i.e., verbs vs. nouns), but may simply be explained by task difficulty or task-specific demands (see Thompson-Schill et al., 1997); in other words, the left inferior frontal gyrus does not appear to be associated with verbs per se, but with the grammatical class requiring the most resources in a specific task (i.e., verbs in the PNT, nouns in the GCST). 


\section{References}

Bedny, M., \& Thompson-Schill, S.L. (2006). Neuroanatomically separable effects of imageability and grammatical class during single-word comprehension. Brain and Language, 98, 127-139.

Crepaldi, D., Aggujaro, S., Arduino, L.S., Zonca, G., Ghirardi, G., Inzaghi, M.G., Colombo, M., Chierchia, G., Luzzatti, C. (2006). Noun-verb dissociation in aphasia: The role of imageability and functional locus of the lesion. Neuropsychologia, 44/1, 73-89.

Fujimaki, N., Miyauchi, S., Putz, B., Sasaki, Y., Takino, R., Sakai, K., \& Tamada, T. (1999). Functional magnetic resonance imaging of neural activity related to orthographic, phonological, and lexico-semantic judgments of visually presented characters and words. Human Brain Mapping, 8, 44-59.

Martin, A., Haxby, J.V., Lalonde, F.M., Wiggs, C.L., \& Ungerleider, L.G. (2005). Discrete cortical regions associated with knowledge of color and knowledge of action. Science, 270, 102-105.

Shapiro, K., Mottaghy, F.M., Schiller, N.O., Poeppel, T.D., Fluss, M.O., Muller, H.W., Caramazza, A., \& Krause, B.J. (2005). Dissociating neural correlate for verbs and nouns. Neuroimage, 24, 1058-1067.

Thompson-Schill, S.L., D'Esposito, M., Aguirre, G.K. and Farah, M.J. (1997). Role of the left inferior prefrontal cortex in retrieval of semantic knowledge. Proceedings of the National Academy of Science, 94, 14792-14797.

Tranel, D., Martin, C., Damasio, H., Grabowski, T.J., \& Hichwa, R. (2005). Effects of nounverb homonymy on the neural correlates of naming concrete entities and actions. Brain and Language, 92, 288-299.

Tyler, L.K., Stamatakis, E.A., Dick, E., Bright, P., Fletcher, P., \& Moss, H.E. (2003).Objects and their actions: evidence for a neurally distributed semantic system. NeuroImage, 18, 542-557. 
Figure 1. Task-by-grammatical-class interaction in both RT and fMRI data; (a) the RTs (ms) indicate that nouns are retrieved faster than verbs in the PNT, whereas verbs are retrieved faster than nouns in the GCST; $(b)$ the same interaction emerges in the fMRI data: the left inferior frontal gyrus, the left insula, the left inferior temporal gyrus and the middle and superior occipital gyrus bilaterally are associated with verbs in the PNT and whit nouns in the GCST.

(a)

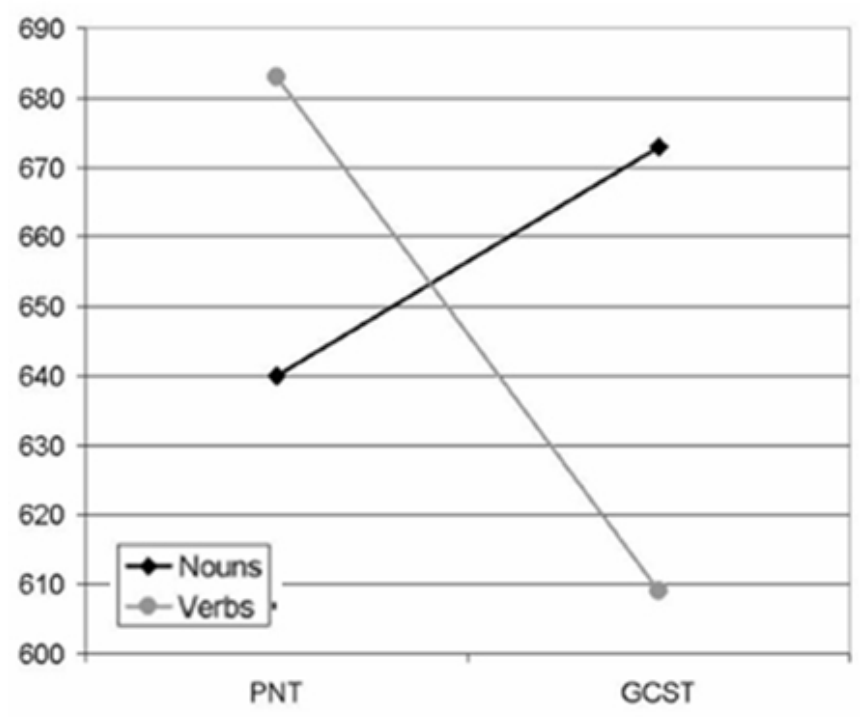

(b)

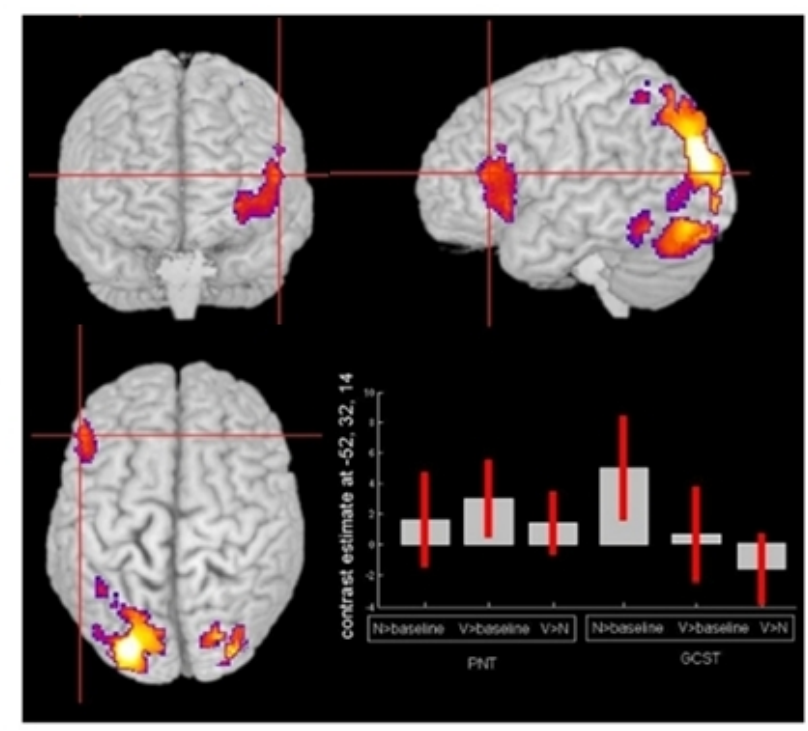

